\title{
Measurements of the muonic component of air showers at the Pierre Auger Observatory
}

\author{
Jeff Allen ${ }^{1, a}$ \\ ${ }^{1}$ New York University, USA
}

\begin{abstract}
Several methods have been developed by the Pierre Auger Collaboration to estimate the muon content of air showers from ultra-high energy cosmic rays. The data of the Pierre Auger Observatory will be compared with predictions based upon EPOS 1.99 and QGSJET-II-3 hadronic interaction models. In addition to the direct measures of the muonic content, the combination of a fluorescence detector and a muon sensitive surface array allows for a direct test of air shower simulations which is sensitive to both the shower core and large distances from the core. These methods reveal a deficit of muons in air shower simulations with proton primaries and that the energy assignment based upon simulations of the surface array signal is systematically higher than that derived from the florescence detector. Summary: I will discuss the deficit in the number of muons currently predicted by simulations when compared to the data of the Pierre Auger Observatory. I will describe the methods used to measure the muon content, including sources of systematic uncertainty, and give their current results. Finally, I will present the Collaborations current understanding of the nature of the discrepancy, which could arise from an energy scale problem, composition, or deficiencies in the hadronic interaction models.

See references $[1,2]$
\end{abstract}

\section{References}

[1] Allen, J. for the Pierre Auger Collaboration, "Interpretation of the signals produced by showers from cosmic rays of $10^{19} \mathrm{eV}$ observed in the surface detectors of the Pierre Auger Observatory". 32nd International Cosmic Ray Conference, Beijing, China. 2011. arXiv:1107.4804.

[2] Yushkov, A. for the Pierre Auger Collaboration, "Measurements of the muon shower content at the Pierre Auger Observatory". International Symposium on Future Directions in Cosmic Ray Physics, CERN, Geneva, 2012.

http://indico.cern.ch/contributionDisplay.py?contribId=88\&sessionId=4\&confId $=152124$

\section{Slides}

The slides of the talk can be found on the website of the symposium ISVHECRI 2012: https://indico.desy.de/conferenceOtherViews.py?view=standard\&confId=4594

\footnotetext{
ajdallen83@gmail.com
} 